\title{
Dark energy and supermassive black holes
}

\author{
Pedro F. González-Díaz \\ Colina de los Chopos, Centro de Física “Miguel A. Catalán,” Instituto de Matemáticas y Física Fundamental, \\ Consejo Superior de Investigaciones Científicas, Serrano 121, 28006 Madrid, Spain
}

(Received 25 February 2004; published 24 September 2004)

\begin{abstract}
This paper deals with a cosmological model in which the universe is filled with tachyon dark energy in order to describe current and future accelerating expansion. We obtain that the simplest condition for the regime of phantom energy to occur in this scenario is that the scalar field be Wick rotated to imaginary values which correspond to an axionic field classically. By introducing analytical expressions for the scale factor or the Hubble parameter that satisfy all constraint equations of the used models we show that such models describe universes which may develop a big rip singularity in the finite future. It is argued that, contrary to a recent claim, the entropy for a universe filled with dark energy is definite positive even on the phantom regime where the universe would instead acquire a negative temperature. It is also seen that, whichever the fate of the tachyonic accelerating universe, it will be stable to any fluctuations of the scalar field, and that since the considered models have all an imaginary sound speed, any overdense regions will undergo an accelerated collapse leading rapidly to formation of giant black holes. Finally the conjecture is advanced that these black holes may be the supermassive black holes that most galaxies harbor at their center.
\end{abstract}

DOI: 10.1103/PhysRevD.70.063530

PACS numbers: 98.80.-k, 98.62.Js

\section{INTRODUCTION}

The discovery that high redshift supernovae of type Ia are less bright than expected $[1,2]$ has opened a really new cosmological scenario with far-reaching implications which may even reach the standard model of particle physics and the very nature of general relativity itself. The straightforward interpretation of this result is that the Universe is currently undergoing a period of accelerated expansion, similar to primordial inflation, but with a rather uncertain future. A most conservative approach to explain the result while keeping general relativity essentially untouched is invoking the inclusion of a now dominating extra component with negative pressure, usually known as dark energy [3], in the Universe. There are several candidates for dark energy whose properties are being probed by cosmological observations, including mainly a pure cosmological constant, quintessence, or k-essence [5] scalar fields (which may [6] or may not be tracked [7]), and some unified dark matter scenarios among which two main plausible models have been suggested: the generalized Chaplygin gas [8], which is described by a highly nonconventional equation of state, and the cosmic tachyon theory based on noncanonical kinetic energy [9].

On the other hand, the increasing dropping of detailed analysis leading to quite an ample observationally acceptable parameter space beyond the cosmological-constant barrier [10] is opening the really intriguing possibility that the universe is currently dominated by what is dubbed as phantom energy [11]. Phantom energy has rather weird properties which include [12]: an energy density increasing with time, naively unphysical superluminal speed of sound, violation of the dominant energy condition which would eventually allow existence of inflating wormholes and ringholes [13], and ultimately emergence of a doomsday singularity in the finite future which is known as the big rip [14]. The regime for phantom energy takes place for state equation parameters $\omega=p / \rho<-1$ and has been shown to occur in all current dark-energy models. However, whereas the big rip singularity is allowed to happen in quintessence [14] and $\mathrm{k}$-essence [15] models, it is no longer present in models based on generalized Chaplygin-gas equations of state $[8,16]$ having the form $P=-A / \rho^{n}$, with $A$ and $n$ being constants. No discussion has been so far made nevertheless on the occurrence of phantom energy and big rip in the other major contender model for unified dark energy: the tachyon matter scenario of Padmanabhan et al. and others $[9,17]$ or its subquantum generalization [18]. Abramo and Finelli have in fact used [19] a BornInfeld Lagrangian with a power-law potential and recovered a nice dark-energy behavior, but did not consider the negative kinetic terms which appear to characterize phantom energy.

While defining a phantom energy regime in such scenarios appears to be rather straightforward, it is quite more difficult to obtain an associated expression for the scale factor of the accelerating universe which allows us to see whether or not a big rip singularity may occur. Under the assumption of a constant parameter for the equation of state, we derive in this paper a rather general solution for the scale factor of a universe dominated by tachyon matter, and show that, quite similarly to as it happens in current quintessence models [2,3,6,7], that solution may predict both an ever expanding accelerating behavior and the occurrence of a big rip singularity, 
theoretically covering therefore all observationally allowed possibilities other than a future big crunch.

Unified dark matter or energy models are very interesting $[8,9]$ in that they are able to simultaneously describe the properties of both dark matter and some form of dark energy (often a cosmological constant) as two opposite limiting situations (at high and low densities, respectively) arising from just one theory. The generalized cosmic Chaplygin models have been however claimed to be observationally inconsistent and actually unstable to short-scale fluctuations [20]. This conclusion was extended to also encompass any unified dark matter or energy models, including the cosmic tachyon scenario [20]. However, that conclusion is true only when both baryons and dark matter are assumed to vanish in the Chaplygin model. If a given, finite fraction of either is introduced in this model then the oscillations may turn out to become very small and do not necessarily spoil the spectra $[21,22]$.

In the present paper we shall use our general solution for the scale factor in a universe filled with tachyon dark energy in order to analyze in some detail the nature and evolution of the fluctuations. We obtain that the unified tachyon theory is stable to any fluctuations and that these may contribute the structure in the universe in a rather decisive way. Actually we conjecture that the necessary accelerated collapse of the formed overdense patches may lead to the formation of the kind of giant black holes which are now believed to occur at the center of most galaxies [23].

The paper can be outlined as follows. In Sec. II we discuss a rather general solution for the scale factor which satisfies all requirements and constraints imposed by tachyon theory. The phantom regime for such a solution is then investigated in this section where it is also seen that it shares all funny properties of quintessential phantom energy, including the new characteristic of having a negative temperature, even though its entropy keeps being definite positive for any value of the equation of state parameter. The evolution of fluctuations with small wavelengths is considered in some detail in Sec. III, using both a synchronous perturbation formalism and the relativistic analog of the Newtonian first order perturbation equation. Sec. IV contains a discussion on the gravitational spherical collapse of formed overdensity regions which is further accelerated by pressure negativeness and the very nature of the tachyonic stuff. The conjecture that the giant black holes formed as a result of that collapse could actually be those black holes which most galaxies harbor at their central bulge is advanced in Sec. V. Results are summarized and briefly discussed in Sec. VI.

\section{TACHYON MODEL FOR DARK ENERGY}

For the most favored cosmological spatially flat scenario, the Friedmann equations read

$$
H^{2} \equiv\left(\frac{\dot{a}}{a}\right)^{2}=\frac{8 \pi G \rho}{3}, \quad \frac{\ddot{a}}{a}=-\frac{4 \pi G(\rho+3 P)}{3},
$$

where $\rho=\rho_{N R}+\rho_{R}+\rho_{\phi}$ is the energy density for, respectively, nonrelativistic, relativistic, and tachyon matter, and $P$ is the corresponding pressure. We shall restrict ourselves to consider a description of the current cosmic situation where it is assumed that the tachyon component largely dominates and therefore we shall disregard what follows the nonrelativistic and relativistic components of the matter density and pressure. For the tachyon field $\phi$ we have [17]

$$
\rho=\frac{V(\phi)}{\sqrt{1-\dot{\phi}^{2}}}, \quad P=-V(\phi) \sqrt{1-\dot{\phi}^{2}},
$$

in which $V(\phi)$ is the tachyon potential energy. Assuming an equation of state $P=\omega \rho$ for the tachyon matter, we then deduce that

$$
\omega=\dot{\phi}^{2}-1
$$

Finally, the equation of motion for $\phi$ is

$$
\ddot{\phi}+\left(1-\dot{\phi}^{2}\right)\left[3 H \dot{\phi}+\frac{1}{V(\phi)} \frac{d V(\phi)}{d \phi}\right]=0 .
$$

\section{A. $\omega>-1$}

We shall show next that there exists a general solution for the scale factor $a(t)$ in this tachyon-field scenario which has exactly the same dependence on time as that has been obtained in the general solution for a pure quintessence scalar field, and hence we also show that such a scenario admits the existence of a tachyon phantom field which leads to a singularity in finite time. In fact, a recipe has been provided by Padmanabhan himself [17] according to which, given the explicit form for the scale factor $a(t)$, a complete specification of the full $\phi$-field theory can be achieved by using the following relations:

$$
\begin{gathered}
\frac{\dot{\rho}}{\rho}=2 \frac{\dot{H}}{H}, \\
\dot{\phi}=\left(-\frac{2}{3} \frac{\dot{H}}{H^{2}}\right)^{1 / 2}, \\
V=\frac{3 H^{2}}{8 \pi G}\left(1+\frac{2}{3} \frac{\dot{H}}{H^{2}}\right)^{1 / 2} .
\end{gathered}
$$

Our task then is to choose a general expression for $a(t)$ which simultaneously satisfies relations (2.5), (2.6), and (2.7), together with the Friedmann Eqs. (2.1) and the equation of motion for the tachyon field (2.4), which will be able to match the accelerating behavior of the current Universe and implies a physically reasonable and suitably motivated field potential. If we assume a linear time dependence of the tachyon field $\phi$ and hence con- 
stancy of parameter $\omega$, then it is not difficult to check that a general form of such an expression for $a(t)$ can be written as

$$
a(t)=\left[a_{0}^{3(1+\omega) / 2}+\frac{3}{2}(1+\omega) t\right]^{2 /[3(1+\omega)]},
$$

where $a_{0}$ is the initial value of the scale factor at the onset of tachyon dark-energy domination. We note that this solution describes an accelerating universe in the interval $-1 / 3>\omega>-1$. At the extreme point $\omega=-1 / 3, a(t)$ describes a universe whose size increases just as $t$, such as it should be expected. It is worth realizing that by simply trivially rescaling the time parameter, solution (2.8) turns out to be nothing but the scale factor that represents the most general solution for the case of a quintessence scalar field for a constant equation of state [13]. On the other hand, for a scale factor (2.8) the tachyon field and potential are given by

$$
\begin{gathered}
\phi=\phi_{0}+\sqrt{1+\omega} t, \\
V(\phi)=\frac{3 \sqrt{-\omega}}{8 \pi G\left[a_{0}^{3(1+\omega) / 2}+\frac{3}{2} \sqrt{1+\omega}\left(\phi-\phi_{0}\right)\right]^{2}} .
\end{gathered}
$$

We note that as $\phi \rightarrow \infty$ this potential reasonably vanishes after taking the form already considered by Padmanabhan and others [17]. As $\phi \rightarrow \phi_{0}$ at $t=0$, $V(\phi)$ tends to be a finite constant value, so clearly separating from the unphysical behavior of the potential considered by Padmanabhan and compatible with what can be supported by string theories [17]. We regard therefore potential (2.10) as being physically reasonable. Finally, using Eqs. (2.2) and (2.3) and (2.8) and (2.9), we obtain for the speed of sound

$$
c_{s}^{2}=\frac{\dot{P}}{\dot{\rho}}=\omega .
$$

This result is in contradiction with the value $c_{s}^{2}=-\omega$ considered in Refs. [20,21]. The reason for that discrepancy comes from the definitions used in this paper of the Lagrangian [17], $L=P=-V(\phi) \sqrt{1-\dot{\phi}^{2}}$, and the energy density given in Eq. (2.2).

For the accelerating-expansion regime, we see thus that the speed of sound becomes imaginary, a case which could imply an accelerating collapse of the tachyon stuff that can still be circumvented however [24]. Equation (2.11) is compatible with all the above requirements, provided we assume a linear time-dependence for the field $\phi$. On the other hand, since $\omega<0$, this equation may be also compatible with a wave equation for tachyon fluctuations where there is factor $-\omega$ in front of the Lagrangian, such as the one used by Frolov, Kofman, and Starobinsky [20].

\section{B. $\omega<-1$}

The phantom energy regime will be characterized by values of the state equation parameter such that $\omega<-1$ and a consequent violation of the dominant energy condition, i.e.,

$$
P+\rho=\frac{V(\phi) \dot{\phi}^{2}}{\sqrt{1-\dot{\phi}^{2}}}<0 .
$$

Such a regime can be obtained by simply Wick rotating the tachyon-field so that $\phi \rightarrow i \Phi$, with which the field $\Phi$ can be viewed as an axion tachyon field [25], as the scale factor $a(t)$ and the field potential $V(\Phi)$ keep being positive and given, respectively, by

$$
a(t)=\left[a_{0}^{-3(|\omega|-1) / 2}-\frac{3}{2}(|\omega|-1) t\right]^{-2 /[3(|\omega|-1)]},
$$

which accounts for a big rip singularity at finite future time

$$
t_{*}=\frac{2}{3(|\omega|-1) a_{0}^{3(|\omega|-1) / 2},}
$$

and

$$
V(\Phi)=\frac{3 \sqrt{|\omega|}}{8 \pi G\left[a_{0}^{-3(|\omega|-1) / 2}-\frac{3}{2} \sqrt{|\omega|-1}\left(\Phi-\Phi_{0}\right)\right]^{2}},
$$

with $\Phi_{0} \rightarrow-i \phi_{0}$. We note that both this potential and the phantom tachyon energy density,

$$
\rho_{\Phi}=\frac{3}{8 \pi G\left[a_{0}^{-3(|\omega|-1) / 2}-\frac{3}{2}(|\omega|-1) t\right]^{2}},
$$

(which has been obtained by using Eqs. (2.2) and (2.3), and (2.15)) increase with time up to blowing up at the singularity at $t=t_{*}$, to steadily decrease toward zero thereafter, in a regime where the Universe contacts to finally vanish as $t \rightarrow \infty$. Thus, the tachyon model for dark energy contains a regime for phantom energy which preserves all the weird properties shown by this in current quintessence and k-essence scenarios; i.e., superluminal imaginary speed of sound, increasing energy density, violation of dominant energy condition, and a big rip singularity followed by a contracting phase.

It has been quite recently pointed out [26] that the entropy for phantom energy is definite negative, and that therefore a cosmic regime with phantom energy should be excluded. However, there exists a rather general argument, valid for all phantom models studied so far including the one considered in this paper, which appears to prevent these conclusions. In fact, for a general Friedman-Robertson-Walker (FRW) flat universe filled with a dark energy satisfying the equation of state $p=$ $\omega \rho$ (with $\omega=$ const.) where the first law of thermody- 
namics holds and the entropy per comoving volume stays constant [27], the temperature of the Universe is given by

$$
\mathrm{T}=\kappa(1+\omega) a^{-3 \omega},
$$

where $\kappa$ is a positive constant. From Eq. (2.17) it is already seen that $\mathrm{T}$ is negative for $\omega<-1$. On the other hand, it is well known that by integrating the cosmic law of energy conservation, $\dot{\rho}+3 \rho(1+\omega) H=0$, we have

$$
\rho=\rho_{0} a^{-3(1+\omega)}
$$

Now, from Eqs. (2.17) and (2.18) one can readily derive the following generalized Stefan-Boltzmann law

$$
\rho=\rho_{0}\left[\frac{\mathrm{T}}{\kappa(1+\omega)}\right]^{(1+\omega) / \omega} .
$$

Note that for $\omega=1 / 3 \mathrm{Eq}$. (2.19) consistently reduces to the usual law for radiation, and that for $0>\omega>-1$ the dark-energy density decreases with the temperature, which is already a rather weird behavior. However, for the regime where $\omega<-1$, in order to preserve $\rho$ positive, we must necessarily take $\mathrm{T}<0$, and therefore $\rho$ will increase with $|\mathrm{T}|$. Since on the phantom regime $\rho$ increases with the scale factor $a(t)$ it also follows that $|\mathrm{T}|$ increases as the universe expands on that regime. Finally, a general expression for the dark-energy entropy can also be obtained [26] which reads

$$
S=C_{0}\left(\frac{\mathrm{T}}{1+\omega}\right)^{1 / \omega} V,
$$

where $C_{0}$ is a positive constant and $V$ is the volume of the considered portion within the dark-energy fluid. It follows that, contrary to the claim in Ref. [26], the entropy of a dark-energy universe is always positive, even on the phantom regime. Actually, by inserting Eq. (2.17) into Eq. (2.20) one attains that $S=$ Const. when we take $V$ as the volume of the entire universe.

It is nonetheless the temperature which becomes negative instead of entropy for $\omega<-1$. Even though it is not very common in physics and therefore can be listed as just another more weird property of the phantom scenario, a negative temperature is not unphysical or meaningless. Systems with negative temperature have already been observed in the laboratory and interpreted theoretically. In the case of phantom energy it means that the entropy of a phantom universe monotonically decreased if one would be able to add energy to that universe. Hence, a $\omega<-1$ universe would always be "hotter" than any $\omega>-1$ universe, and if two copies of the Universe were taken, one with positive and other with negative temperature, and put them in thermal contact, then heat would always flow from the negative-temperature universe into the positive-energy universe. It could yet be argued that negative temperature is a quantum-statistical mechanics phenomenon and therefore cannot be invoked in the classical realm. However, a negative temperature given by
Eq. (2.17) when $\omega<-1$ [27] can still be heuristically interpreted along a way analogous to how, e.g., black hole temperature can be interpreted (and derived) without using any quantum-statistical mechanics arguments; that is by simply Wick rotating time, $t \rightarrow i \tau$, and checking that in the resulting Euclidean framework $\tau$ is periodic with a period which precisely is the inverse of the Hawking temperature [28]. Thus, the Euclideanized black hole turns out to be somehow "quantized". Similarly in the present case, the phantom regime can be obtained by simply Wick rotating the classical scalar field, $\phi \rightarrow i \Phi$, which, by Eq. (2.9), is equivalent to rotating time so that $t \rightarrow i \tau$, too. It is in this sense that the phantom fields are also quantized and that the emergence of a negative temperature in the phantom regime becomes consistent.

\section{RENAISSANCE OF THE UNIFIED TACHYON DARK MATTER MODEL}

The biggest problem which has been claimed to be confronted by tachyon theory of dark energy refers to its property of being a unified dark matter model with a nonzero speed of sound. In fact such a kind of model has been ruled out as causing violently unphysical blowup in the matter power spectrum [20]. Actually, the precise way in which the unified dark matter model separates from what is observed has been only carried out for the case of the so-called cosmic generalized Chaplygin gas [20] whenever baryons and dark matter are assumed to be absent. Rather general arguments were then raised [20] to generalize the conclusion to any unified dark-energy model whose equation of state allows for nonzero values of the sound speed. In what follows we shall show that, even though baryons and dark matter are not explicitly introduced, such a generalization is no longer valid, at least for unified models of tachyon dark matter and energy. We will in fact see that perturbations with wavelength below the Jeans scale do not increase along the available time interval for the imaginary values taken by the sound speed for the tachyon field for any $\omega$ and a scale factor as given by Eq. (2.8). The stress tensor for the tachyon scalar field can be written in the perfect fluid form [17]

$$
T_{k}^{i}=(p+\rho) u^{i} u_{k}-p \delta_{k}^{i}
$$

where the pressure and energy density are given in Eq. (2.2) and

$$
u_{k}=\frac{\partial_{k} \phi}{\dot{\phi}} .
$$

This stress tensor can be now split into a pressureless, dark matter component and a dark-energy component [17]: $\rho=\rho_{v}+\rho_{\mathrm{DM}}$ and $p=p_{v}+p_{\mathrm{DM}}$. Now, the vacuum component can correspond to either a cosmological constant if we choose [17] 


$$
\begin{gathered}
\rho_{\mathrm{DM}}=\frac{V(\phi) \dot{\phi}^{2}}{\sqrt{1-\dot{\phi}^{2}}}, \quad p_{\mathrm{DM}}=0, \\
\rho_{v}=V(\phi) \sqrt{1-\dot{\phi}^{2}}, \quad p_{v}=-\rho_{v},
\end{gathered}
$$

(for which $\omega_{v}=-1$ ), or to a slowly-varying quintessential scalar field when we choose

$$
\begin{gathered}
\rho_{\mathrm{DM}}=V(\phi) \sqrt{1-\dot{\phi}^{2}}, \quad p_{\mathrm{DM}}=0, \\
\rho_{v}=\frac{V(\phi) \dot{\phi}^{2}}{\sqrt{1-\dot{\phi}^{2}}}, \quad p_{v}=-V(\phi) \sqrt{1-\dot{\phi}^{2}} .
\end{gathered}
$$

In this latter case we have

$$
\omega_{v}=1-\dot{\phi}^{-2}
$$

which for sufficiently slowly-varying fields can even take on values $<-1$.

\section{A. Fluctuation formalism}

Using the synchronous perturbation formalism first developed by Lifshitz and Khalatnikov, the study of scalar perturbations, $h$, around the FRW metric can be reduced to investigate the solutions of the equation for the $k$ th mode [29]

$$
\mu^{\prime \prime}+\left(k^{2} c_{s}^{2}-\frac{a^{\prime \prime}}{a}\right) \mu=0,
$$

where $I=d / d \eta$, with $\eta=\int d t / a$ being the conformal time, $c_{s}^{2}=$ const. and the function $\mu$ is defined as

$$
\mu=\frac{\left(h^{\prime}+\alpha \gamma h\right) a}{\alpha \sqrt{\gamma} c_{s}}=\frac{i(\dot{H} h-H \dot{h}) a}{H \sqrt{\dot{H}} c_{s}},
$$

with $\quad \cdot=d / d t, \quad \alpha=a^{\prime} / a=\dot{a}, \quad \gamma=1-\alpha^{\prime} / \alpha^{2}=$ $1-\ddot{a} / \dot{a}^{2}$, the scale factor $a(t)$ being given by Eq. (2.8) and

$$
a(\eta)=\left[\frac{(1+3 \omega) \eta}{2}\right]^{2 /(1+3 \omega)},
$$

where

$$
\eta=\frac{2}{1+3 \omega} a(t)^{(1+3 \omega) / 2} .
$$

At first sight it seems that the small-scale fluctuations must grow when $c_{s}^{2}<0$. In fact, taking the limit for large $k$ in Eq. (3.8) we obtain that the solution should be a growing mode for $c_{s}^{2}<0$. But this is a growing mode in the conformal time $\eta$, not in the physical time $t$, with $\eta$ and $t$ being related to each other by means of Eq. (3.11). It can be readily seen by using Eq. (2.8) that a growing mode in $\eta$ necessarily implies a decreasing mode in $t$ for any $\omega<-1 / 3$ characterizing an accelerating universe.
We note, moreover, that the function $\mu$ is pure imaginary for $c_{s}$ real and real for pure imaginary $c_{s}$. In the present model, we have from Eq. (2.11) that $c_{s}^{2}=\omega$, thus, if $c_{s}^{2}=$ $\omega$ is assumed to be constant, all the problem reduces to study a simple differential equation given by

$$
\mu^{\prime \prime}+\left[k^{2} c_{s}^{2}-\frac{2\left(1-3 c_{s}^{2}\right)}{\left(1+3 c_{s}^{2}\right)^{2} \eta^{2}}\right] \mu=0 .
$$

If the range of equation of state parameter would be extended to include the regime $0<\omega<1 / 3$, then in that regime all would happen like in the usual case, that is the perturbations would oscillate for $k^{2} c_{s}^{2}>>2(1-$ $3 \omega) /\left[(1+3 \omega)^{2} \eta^{2}\right]$ and had an exponential behavior for $k^{2} c_{s}^{2}<<2(1-3 \omega) /\left[(1+3 \omega)^{2} \eta^{2}\right]$. However, if we extend the range of $\omega$-values to also cover values $\omega>1 / 3$, then gravity would behave like a repulsive force and play on the same team as pressure does. For the most interesting range where the universe shows an acceleratingexpansion, $\omega<-1 / 3$, however, it is the pressure what plays on the same team as gravity does. In that case, if $k^{2} c_{s}^{2}>>2(1-3 \omega) /\left[(1+3 \omega)^{2} \eta^{2}\right]$, then the solution to the differential perturbation equation reads

$$
\mu \propto e^{\left(k\left|c_{s}^{2}\right|^{1 / 2} \eta\right)}=\exp \left( \pm k\left|c_{s}^{2}\right|^{1 / 2} \frac{2 T^{(1+3 \omega) /[3(1+\omega)]}}{1+3 \omega}\right),
$$

with

$$
T=a_{0}^{3(1+\omega) / 2}+\frac{3}{2}(1+\omega) t .
$$

In the range of state equation parameters $-1 / 3>\omega=$ $c_{s}^{2}>-1$ it can be seen that $\mu$ decreases with time $t$, from an initial value

$$
\mu=\mu_{0} \propto \exp \left[\frac{ \pm 2 k\left|c_{s}^{2}\right|^{1 / 2}}{1+3 c_{s}^{2}} a_{0}^{\left(1+3 c_{s}^{2}\right) / 2}\right],
$$

at $t=0$, to become a minimum $\mu \propto 1$, as $t \rightarrow \infty$. Also for the case where $\omega=c_{s}^{2}<-1 \mu$ starts with a value given by Eq. (3.14) and becomes $\propto 1$ as one approaches the big rip time $t_{*}$. That decrease of density fluctuations with small wavelengths clearly separates from what was previously predicted by Carturan and Finelli, and Sandvik et al. [20], being also in sharp contrast with what arises from the equation that describes the evolution of small perturbations in Newtonian physics, that is [30],

$$
\ddot{\boldsymbol{\epsilon}}_{1}+\left(k^{2} c_{\ell}^{2}-4 \pi G \epsilon_{0}\right) \epsilon_{1}=0,
$$

where $c_{\ell}^{2}=p_{1} / \epsilon_{1}$, with $p_{1}$ and $\epsilon_{1}$ being the pressure and energy density first order perturbations. In fact, for $c_{\ell}^{2}<$ 0 , it is obtained that $\epsilon_{1}$ increases exponentially with time so that

$$
\epsilon_{1} \propto \exp \left( \pm \sqrt{k^{2}\left|c_{\ell}^{2}\right|+4 \pi G \epsilon_{0}} t\right)
$$


Although the mathematical reason for having obtained such a different behavior in our estimate for the time evolution of density perturbations must be addressed to the specific form with which the scale factor used in this paper depends on $\omega$ (which generalizes more restricted possible expressions), the physical reason why the shortscale fluctuations are stable in our model, even in the case where no contribution from baryons and dark matter is explicitly considered, can be ultimately interpreted to be the unified character of that model where dark matter is also implicitly present.

We shall turn next to derive general analytical solutions for the density contrast, $\delta$, in our tachyon model, thereby checking and refining the conclusions attained so far in the above heuristic model. That study will allow us, moreover, to deal in a proper way with the important issue of the initial conditions for fluctuations. If we assume that the tachyon field is given by Eq. (2.9), the tachyon-field theory considered in Sec. II is fully equivalent to the simple theory of a perfect fluid with constant equation of state $P=\omega \rho$ and squared sound speed $c_{s}^{2}=$ $\omega$ in a flat universe with a scale factor given by either Eq. (2.8) if $\omega>-1$ or Eq. (2.13) if $\omega<-1$. Thus, even though the density fluctuations of a perfect fluid generally behave differently from the density fluctuations of a scalar field, in an adiabatic approach we can use the relativistic analog of the Newtonian first order perturbation equation in order to describe fluctuations of the density contrast. In our present case, from the form of the energy density and the scale factor one can derive an expression for the relativistic analog of the Newtonian first-order perturbation equation in Fourier space [31] which reads for a density contrast perturbation $\delta_{k}$ with wave vector $k$

$$
\begin{gathered}
\delta_{k}^{\prime \prime}+\frac{1}{2}\left(1-9 c_{s}^{2}\right) \delta_{k}^{\prime}- \\
{\left[\frac{3}{2}\left(1+2 c_{s}^{2}-3 c_{s}^{4}\right)-\left(\frac{k c_{s}}{H a}\right)^{2}\right] \delta_{k}=0,}
\end{gathered}
$$

where now $I=d / d \ln a$ and we have used $\omega=c_{s}^{2}$. Because of the presence of a first-order derivative term in Eq. (3.17), also in this case the taking of the simple large $k$ limit in this equation when $c_{s}^{2}<0$ cannot by itself uncover the real behavior of small-scale fluctuations with physical time $t$. An explicit integration of this differential equation should also be done. For this to be accomplished, one can now rewrite Eq. (3.17) in the form

$$
\begin{gathered}
a^{2} \delta_{k}^{\prime \prime}+\frac{3}{2} a\left(1-3 c_{s}^{2}\right) \delta_{k}^{\prime}- \\
{\left[\frac{3}{2}\left(1+2 c_{s}^{2}-3 c_{s}^{4}\right)-k^{2} c_{s}^{2} a^{1+3 c_{s}^{2}}\right] \delta_{k}=0,}
\end{gathered}
$$

where now $I=d / d a$ and we have used Eq. (2.8). Since $c_{s}^{2}<0$ this differential equation admits a general analytical solution which can be expressed in terms of modified Bessel functions [32]. Depending on the initial conditions that solution can be taken to be either some of the following ones or some of their combinations

$$
\begin{gathered}
\delta_{k}=a^{-\left(1-9 c_{s}^{2}\right) / 4} e^{\left[i \pi\left(5+3 c_{s}^{2}\right)\right] /\left[4\left(1+3 c_{s}^{2}\right)\right]} I_{\left(5+3 c_{s}^{2}\right) /\left[2\left(1+3 c_{s}^{2}\right)\right]\left( \pm \frac{2 k\left|c_{s}^{2}\right|^{1 / 2}}{1+3 c_{s}^{2}} a^{\left(1+3 c_{s}^{2}\right) / 2}\right)} \\
\delta_{k}= \pm \frac{2}{i \pi} a^{-\left(1-9 c_{s}^{2}\right) / 4} e^{\left[i \pi\left(5+3 c_{s}^{2}\right)\right] /\left[4\left(1+3 c_{s}^{2}\right)\right]} K_{-\left(5+3 c_{s}^{2}\right) /\left[2\left(1+3 c_{s}^{2}\right)\right]}\left( \pm \frac{2 k\left|c_{s}^{2}\right|^{1 / 2}}{1+3 c_{s}^{2}} a^{\left(1+3 c_{s}^{2}\right) / 2}\right)
\end{gathered}
$$

for $-1 / 3>c_{s}^{2}>-5 / 3$,

$$
\delta_{k}= \pm \frac{2}{i \pi} a^{-\left(1-9 c_{s}^{2}\right) / 4} e^{-\left[i \pi\left(5+3 c_{s}^{2}\right)\right] /\left[4\left(1+3 c_{s}^{2}\right)\right]} K_{\left(5+3 c_{s}^{2}\right) /\left[2\left(1+3 c_{s}^{2}\right)\right]}\left( \pm \frac{2 k\left|c_{s}^{2}\right|^{1 / 2}}{1+3 c_{s}^{2}} a^{\left(1+3 c_{s}^{2}\right) / 2}\right)
$$

for $c_{s}^{2} \leq-5 / 3$.

We must now set the initial conditions for these perturbations. If we assume that dark energy is present in the Universe from its very origin at $a=0$, the simplest and most natural condition that such perturbations should satisfy is that they ought to tend to vanishing values as one approaches the initial singularity both for $\omega>-1$ and $\omega<-1$. One should then set $\delta_{k}=0$ as $a \rightarrow 0$. In the limit $a \rightarrow 0$ (i.e., for arbitrarily large values of the argument $z$ of the modified Bessel function, $B( \pm z)$ ), we have for $B( \pm z)=I( \pm z)$

$$
\delta_{k} \propto a^{-\left(1-3 c_{s}^{2}\right) / 2} \exp \left( \pm \frac{2 k\left|c_{s}^{2}\right|}{1+3 c_{s}^{2}} a^{\left(1+3 c_{s}^{2}\right) / 2}\right)
$$

which vanishes for the + sign (i.e., for $-\pi<\arg z \leq$ $\pi / 2$ ) and blows up for the $-\operatorname{sign}($ i.e., for $\pi / 2<\arg z \leq$ $\pi)$, and for $B( \pm z)=K( \pm z)$,

$$
\delta_{k} \propto a^{-\left(1-3 c_{s}^{2}\right) / 2} \exp \left(\mp \frac{2 k\left|c_{s}^{2}\right|}{1+3 c_{s}^{2}} a^{\left(1+3 c_{s}^{2}\right) / 2}\right),
$$

which vanishes for the lower sign and blows up for the upper sign. Then the above initial conditions imply that 
we have to choose the modified Bessel functions $I(+z)$ and $K(-z)$, or some combination of them, for universes which start being dominated by dark energy at $a=a_{0}$. Note that at $a=a_{0}$, i.e., at the time when dark energy starts to dominate, $\delta_{k}$ consistently takes on finite, nonzero values. It follows that the Universe is stable under perturbations $\delta_{k}$. Moreover, if the initial value of the perturbation at $a=a_{0}$ is small enough then the fluctuation will first grow up to a given maximum value to be steadily damped thereafter, vanishing as $a \rightarrow \infty$, both for $\omega>-1$ and $\omega<-1$. There would then be an interval of scale factor values where these fluctuations had observable effects. Since the sound speed is pure imaginary in all the considered cases, fluctuations will gravitationally collapse in an accelerated fashion to form, together with observable matter, some structures with cosmological interest.

We note finally that consistency of using Eq. (3.17) with the above initial conditions is manifested, if not guaranteed, by the feature that they lead to exactly the same conclusions as those obtained from the synchronous model. A caveat is worth mentioning however. Once we have discussed fluctuations in the linear phase, it appears most convenient to consider the fluctuations in the nonlinear phase, as these could become eventually dominant in some regimes. Such a study is left for a future work.

On the other hand, another caveat should also be mentioned. Even though our solutions apply to scales in the subhorizon regime, if the Universe is expanding in an accelerating fashion then it would also be natural to consider that sooner or later all scales will become larger than the horizon so that the Newtonian approximation would break down, especially when the limit $t \rightarrow \infty$ is approached for $\omega>-1$. In the case that $k^{2} c_{s}^{2}<<2(1-$ $3 \omega) /\left[(1+3 \omega)^{2} \eta^{2}\right]$, the solutions to, for example, Eq. (3.12) can be approximated to

$$
\mu=A \eta^{2 /\left(1+3 c_{s}^{2}\right)}+B \eta^{-\left(1-3 c_{s}^{2}\right) /\left(1+3 c_{s}^{2}\right)},
$$

with $A$ and $B$ arbitrary constants. We then see that for $c_{s}^{2}<-1 / 3, \mu$ will grow with time $t$.

\section{B. Fluctuations in the contracting phase}

Once we have checked that our tachyon model for an accelerating universe is stable against density fluctuations both when $\omega>-1$ and for $\omega<-1$ up to the big rip time $t_{*}$, we next proceed to briefly consider what happens with such fluctuations when $\omega<-1$ at times $t>t_{*}$. This study is of some interest for the following reason. Even though the big rip corresponds to a curvature singularity that does not strictly allow for causal connections between the regions before and after the big rip, this might still be circumvented by connections between these regions rendered physically plausible by means of wormholes inflated by the accelerated expansion [13] or phantom energy accretion [33]. It can be seen however that if we restrict ourselves to the case $\omega<-1$ in Eq. (3.13) then for $t>t_{*}$ there will be a huge growth of the density of perturbations with small wavelengths, so rendering the evolution of the Universe after the big rip fully unstable. On the other hand, in order for studying the evolution of the fluctuations after $t_{*}$ in some more detail, one must introduce the condition that $\delta_{k}=0$ as $t \rightarrow t_{*}$ (i.e., as $a \rightarrow$ $\infty$ ). Now, for $a \rightarrow \infty$ (i.e., as $z$ tends to zero), we have

$$
\begin{aligned}
& \delta_{k}[I( \pm z)] \propto a^{1+3 c_{s}^{2}} \rightarrow 0, \\
& \delta_{k}[K( \pm z)] \propto a^{1+3 c_{s}^{2}} \rightarrow 0, \\
& \text { if }-1>c_{s}^{2}>-5 / 3, \text { and } \\
& \delta_{k}[K( \pm z)] \propto a^{-3\left(1-c_{s}^{2}\right) / 2} \rightarrow 0,
\end{aligned}
$$

for $c_{s}^{2} \leq-5 / 3$. We have to choose then the modified Bessel functions $I(-z)$ and $K(-z)$, or some given combination of them, for universes starting to evolve just after the big rip singularity. It can be then checked that such conditions also predict that the contracting universe that starts evolving just after the big rip is unstable to the considered fluctuations.

\section{STRUCTURE FORMATION IN A TACHYON DARK-ENERGY UNIVERSE}

In this section we will briefly consider the fate of the fluctuations $\delta_{k}$ once they have formed and evolved to their nearly largest size. From the onset of the epoch where dark energy starts dominating the formed overdense regions in the Universe will follow an evolution which is governed by stuff characterized by negative pressure and speed of sound squared. That will inexorably make the force involved in such fluctuations to be attractive as pressure will now contribute on the same attractive pattern as gravity. Thus, the overdense regions would immediately start to undergo an accelerated gravitational collapse which involved both dark matter and dark energy, and hence any now subdominant possible visible matter that could be more or less associated with them, towards a central singularity with infinite density. In practice, since the stuff making up tachyon dark matter energy is expected to be spinless and chargeless, in the present case only some macroscopic dissipative processes, other than pressure, spin degeneracy, or electric charge repulsion, could intervene to convert kinetic energy of collapse into random motions before the singularity. Black holes mainly made from dark matter could be thereby most easily formed at the end of the collapse, though the residual dissipative forces could still stop the collapse before reaching the final dynamical equilibrium.

\section{A. Accelerated spherical collapse}

We shall assume in what follows a spherical collapse model with no shell crossing, so that we can ignore the 
spatial dependence of the involved fields [34]. In principle, the evolution of a spherical overdense patch with scale radius $R(t)$ containing independent dark matter (with density $\rho_{\mathrm{DM}}$ ) and dark energy (with density $\rho=$ $p / \omega)$ is governed by the Raychaudhuri equation [30]

$$
3 \ddot{R}=-4 \pi G R\left[\rho_{\mathrm{DM}}+(1+3 \omega) \rho\right] .
$$

For our unified model, the first term in squared brackets of this equation becomes redundant because dark matter is already contained in the unified tachyon dark description encapsulated in the term containing density $\rho$. Thus, for the case being considered, Eq. (4.1) reduces to:

$$
3 \ddot{R}=-4 \pi G R(1+3 \omega) \rho .
$$

Now, in order to check consistency of our unified darkenergy model, let us first apply Eq. (4.1) to an energy density as given in Eq. (2.2) which, when expressed in terms of variable $T=a_{0}^{3(1+\omega) / 2}+3(1+\omega) t / 2$, reads

$$
\rho=\frac{3}{8 \pi G T^{2}} \text {. }
$$

That is, one is considering the Universe as a whole as a perturbation and, therefore, one should recover the scale factor (2.8) as a solution from Eq. (4.1). This is in fact the case, but we obtain also another solution given by

$$
R=a_{c}=T^{(1+3 \omega) /[3(1+\omega)]}=a(t)^{(1+3 \omega) / 2} .
$$

Note that this additional solution will vanish as $a \rightarrow \infty$ both for $\omega>-1$ and for $\omega<-1$ and represents therefore a collapsing universe.

For a spherical overdense patch with scale radius $R(t)$ within the Universe, the Raychaudhuri equation will be

$$
3 \ddot{R}=-4 \pi G R(1+3 \omega)(\rho+\mu),
$$

where $\rho$ is given by Eq. (4.3) and we have restricted ourselves to consider perturbations of the energy density that correspond only to small wavelengths; that is perturbations that can be described by

$$
\begin{aligned}
\mu & =\mu_{0} \exp \left[\frac{-2 k\left|c_{s}^{2}\right|^{1 / 2} T^{(1+3 \omega) /[3(1+\omega)]}}{1+3 \omega}\right] \\
& =\mu_{0} \exp \left(\frac{-k\left|c_{s}^{2}\right|^{1 / 2} a_{c}}{1+3 \omega}\right) .
\end{aligned}
$$

Obtaining an exact analytical solution to the differential Eq. (4.7) is very difficult and we will simply consider in what follows approximate asymptotic solutions when $T$ is very large or very small, for particular values of $\omega$. On the regime of $\omega>-1$ and $T$ large, we take the observationally plausible value $\omega=-13 / 15$ so that the differential Eq. (4.7) can then be approximated to

$$
R^{\prime \prime}-\frac{128 \pi G}{153}\left(\mu_{0}+\frac{3}{8 \pi G T^{2}}\right) R \simeq 0 .
$$

A solution is then again given in terms of the modified
Bessel function $\wp[32]$

$$
R \simeq T^{1 / 2} \wp \sqrt{53 / 180}\left(\sqrt{\frac{128 \pi G \mu_{0}}{135}} T\right) .
$$

For small values of $T$ on the phantom regime at the particular case that $\omega=-5 / 3$, the approximate differential Eq. (4.7) will become

$$
R^{\prime \prime}-\frac{64 \pi G}{27}\left(\mu_{0}+\frac{3}{8 \pi G T^{2}}\right) R \simeq 0,
$$

which again admits an approximate solution in terms of modified Bessel functions $\wp$, that is [32]

$$
R \simeq T^{1 / 2} \wp \sqrt{41 / 36}\left(\sqrt{\frac{64 \pi G \mu_{0}}{27}} T\right) .
$$

Choosing now for $a_{0}$ a sufficiently large value then $T$ will always be large enough for $\omega>-1$ and small enough for $\omega<-1$. Since the most natural and sufficient initial condition in the present case is that $R<<a$ initially, then the only solutions which satisfy the above initial condition are for $\omega=-13 / 15$ (and generally for any $\omega>-1)$ the one with $\wp=K$, i.e.,

$$
R \propto \exp \left(-\sqrt{\frac{128 \pi G \mu_{0}}{135}} T\right),
$$

and for $\omega=-5 / 3$ (and generally for any $\omega<-1$ ) the one with $\wp=I$, i.e.,

$$
R \propto T^{(1 / 2)(1+\sqrt{41 / 9})} .
$$

Now these two solutions tend to vanish as one lets $a(t) \rightarrow$ $\infty$, so indicating a complete accelerated gravitational collapse of the overdense patches, which can be modulated by the residual dissipative forces. Although the possibility that the black holes that eventually formed as the final state of such collapses actually be those supermassive black holes ultimately driving formation of the observed galaxies $[23,35]$ is just a conjecture we want to advance here (see Sec. V); it already appears interesting to consider that possibility as a quite promising way through which the studied fluctuations may show up observationally.

\section{B. Loss of dark energy}

On the other hand, dark energy does not necessarily follow the collapse of dark matter inside the overdensity regions and therefore there could be an energy loss of dark-energy $\Gamma$ in these regions. In the present case this energy loss can be described by the equation [36]

$$
\Gamma=\dot{\phi} \ddot{\phi}+\left(1-\dot{\phi}^{2}\right)\left(3 \frac{\dot{R}}{R} \dot{\phi}^{2}+\frac{\dot{V}}{V}\right),
$$

with $R$ the scale radius of an average overdense region, and $\phi$ and $V=V(\phi)$ the scalar field and the potential on 
that region. There are important technical difficulties which would appear if we try to calculate $\Gamma$ using the expressions of $R$ discussed in the precedent subsection. In order to evaluate on which cases $\Gamma \neq 0$ we tentatively shall simply interpret for a moment the collapsing solution $a_{c}=T^{(1+3 \omega) /[3(1+\omega)]}$ to the Raychaudhuri equation as though it represented the scale radius of an overdensity patch, i.e., $R=a_{c}$, and then use the two ansätze considered in Sec. IV for the partition of the full energy density and pressure of the unified model into dark matter and dark-energy components. In such a toy description we have for the not partitioned theory

$$
\begin{gathered}
H=\frac{\dot{R}}{R}=\frac{1+3 \omega}{2 T}, \\
\dot{\phi}=\sqrt{\frac{2(1+\omega)}{1+3 \omega}}, \\
V=\frac{3(1+3 \omega)^{3 / 2}(\omega-1)^{1 / 2}}{32 \pi G T^{2}} .
\end{gathered}
$$

Since no partition of energy density and pressure has been made yet, the insertion of Eqs. (4.14) and (4.15) and (4.16)into Eq. (4.13) consistently leads to the result that there is no loss of any energy on the overdense region and $\Gamma=0$. The use of the first ansatz for partitioning dark energy and pressure given by Eq. (3.4) leads to $\omega_{v}=-1$, $\dot{\phi}_{v}=0, H=-T^{-1}$, and $V=3 /\left(4 \pi G T^{2}\right)$, and hence again $\Gamma=0$, as it was also expected for a cosmological constant. However, using the partition given by Eq. (3.6), we obtain $\omega_{v}=1-\dot{\phi}^{-2}$, and finally

$$
\Gamma=\frac{3 \omega}{2 T}\left(\frac{2 \omega^{2}+3 \omega-1}{1-\omega}\right),
$$

which shows a loss of dark-energy in the overdense region for any $\omega>(3+\sqrt{17}) / 4$. We then notice that the collapse tachyonic overdense patches will mainly affect the dark matter component only if the dark-energy component does not correspond to a positive cosmological constant with $\omega_{v}=-1$. For in that case the collapse would equally affect both components.

\section{A CONJECTURE ON GALAXY FORMATION}

Having shown that the evolution of density perturbations in the unified tachyon theory does not lead to a catastrophic growth of perturbations with small wavelengths, but to a negative-pressure-assisted super accelerated gravitational collapse of the dark matter and energy involved in the overdense regions, leaving rapidly formed black holes which may have a large mass, one should now consider the observational consequences from the formation of such holes. At first sight, the results obtained in the present paper could in fact be disregarded under the claim that no giant black holes of the kind discussed in it have been hitherto observed. We are going now to conjecture nevertheless that it is precisely a family of rapidly forming huge black holes with masses analogous to those of the black holes resulting from the accelerated collapse of overdense regions in tachyon unified dark energy discussed in this paper which have been discovered in recent years at the center of most-possibly all - quasars (QSOs) and current luminous galaxies [23]. The rationale supporting such a conjecture runs up along the following points.

1 The idea that bright galaxies may harbor supermassive black holes (SMBHs) actually dates back to the year 1969 and was first advanced by Lynden-Bell [37]. Today it is a rather common belief that mostif not all-galaxies have a central SMBH in their bulge [23]. It is also currently thought that at the time of the formation of one galaxy its present central black hole started also to be formed [36].

2 The way in which SMBHs were formed is still unclear. They might have a primordial origin, be formed from population III stars and their resulting merging, or otherwise [38]. The physical process involved could, respectively, be either by directly collapsing from external pressure in the first instant of big bang or by slow accretion of matter starting from a stellar size, or otherwise. The idea that SMBHs may have passed through an intermediate observable mass stage along a very long accreating process is now gaining increasing support [39].

3 The epoch of galaxy formation is thought to be very broad, extending from $z \sim 20$ to $z \sim 2$. The galaxies were formed from perturbations created at $z \sim 1400$. However, galaxies with redshifts $\geq 2-3$ are not directly observable. Since a baryonic gas inside a dark matter potential collapses and forms stars, any resulting massive stars rapidly evolving and feedbacking heavy elements into the gas, the production of first heavy elements can be approximately equated to the epoch of bright galaxy formation. Metal lines have been so far identified in QSO spectra [40] indicating that, prior to $z=2.5-3$, the heavy metal line strengths are fairly low, with the largest strengths appearing within the interval $z=1.5-$ 2.5. Thus, non luminous galaxies could have existed from nearly the primordial epoch, but the formation of a sufficiently high density of stars in them making these galaxies bright appears to be fairly recent.

4 The observational discovery that there exists a simple relation between the mass of central SMBHs and the speed or the sigma of the stars in the surrounding galaxy bulges [41], which was anticipated by the Silk-Rees theory [42], seems then to indicate that most of such central SMBHs reached-or were created with - their present huge maturity mass just at around $z \sim 2-3$, nearly at, or shortly before, the 
epoch when the giant black holes discussed in the present paper probably started to be collapsed in an accelerated way. The onset of that epoch should nevertheless be marked by the farthest supernova at $z=1.7$ [43], that is to say around $z \sim 1$. Moreover, there exist QSOs at $z \sim 6$ and bright galaxies at $z \sim 3$ which must harbor a SMBH at their central bulge. At first sight, these features would prevent SMBHs formed by the collapse of overdense tachyon dark-energy regions to be suitable candidates for being the central holes that would induce bulge star creation and hence bright galaxy formation. Notwithstanding, there are at least two main arguments against that conclusion. On the one hand, it has been shown [44] that in the presence of a cosmological constant the galaxy formation epoch must have started much later and lasted much longer, possibly all the way to the present. Similarly, taking into account the Friedmann equation for flat geometry, if instead of the cosmological-constant density $\lambda_{0}=\Lambda /\left(3 H_{0}^{2}\right)$ of Ref. [44], we introduce the dynamical dark-energy density considered in this paper, then a theoretical onset for bright galaxy formation can be derived by replacing $\lambda_{0}$ in the formula provided in that reference for the quantity $8 \pi G \rho /\left(3 H_{0}^{2}\right)$. Using then Eqs. (2.2) and (2.3), and (2.10) one can finally have

$$
1+z_{\mathrm{GF}}=\left[H_{0}^{2} T^{2} \Omega_{0} \sinh ^{2}\left(\frac{3 t_{\mathrm{GF}}}{2 T}\right)\right]^{-1 / 3},
$$

where $H_{0}$ is the current value of the Hubble constant and $t_{\mathrm{GF}}$ is the galaxy formation time, $t_{\mathrm{GF}}=t_{d}+t_{c}$, with $t_{d}$ the time needed for a galaxy to start forming stars once the region with local Hubble parameter $H^{\prime}$ has collapsed, and $t_{c}$ is the collapse time which is in the present case determined by

$$
t_{c}=2 \int_{0}^{x_{\max }}\left(U+\frac{2}{x}+\frac{x^{2}}{T^{2}}\right)^{-1 / 2} d x,
$$

where if the mass of the overdense region of scale radius $r$ is denoted by $m$,

$$
\begin{gathered}
x=r(G m)^{-1 / 3}, \\
U=4^{1 / 3}\left[\left(\frac{H^{\prime}}{H}\right)^{2}-\Omega(1+\bar{\delta})-\frac{1}{H^{2} T^{2}}\right]\left(\frac{1+\bar{\delta}}{\Omega H^{2}}\right)^{-2 / 3}
\end{gathered}
$$

and

$$
U+\frac{2}{x_{\max }}+\frac{x_{\max }}{T^{2}}=0,
$$

with $\Omega=\bar{\rho} /\left(3 H^{2}\right), \bar{\rho}$, and $H$ respectively being the mean matter density and Hubble parameter of the Universe. We can check that also in this case for suitable initial values of the observable density con- trast $\bar{\delta}_{i}$, the galaxy formation epoch is shifted toward later times by some two $z$-units if currently $8 \pi G \rho /\left(3 H_{0}^{2}\right) \simeq \Lambda /\left(3 H_{0}^{2}\right) \simeq 0.8$, while it lasts quite longer, possibly all the way to the present, too, such as it occurred in the presence of a positive cosmological constant [44], except in that in the present theory $z_{\mathrm{GF}}$ depend on time $t$ through the parameter $T$. It is worth noticing, e.g., that in the phantom regime where $\omega<-1$, as one approaches the big rip at $T=0, t_{c} \rightarrow 0$, and $z_{G F} \rightarrow 0$, so that, consistent with the ripping off of all materials in the Universe, observers would detect no galaxies at all.

On the other hand, and even more importantly, if a direct interaction term between the two components $\rho_{v}$ and $\rho_{D M}$ transferring energy from one another is assumed to exist and have the form

$$
\begin{gathered}
\gamma=-3 \ell_{P} \sqrt{\Omega_{v}} \omega \rho_{D M}, \quad \Omega_{v}=\frac{\rho_{v}}{3 H^{2}}, \\
\ell_{P}^{2}=\frac{8 \pi G}{3},
\end{gathered}
$$

then it can be readily seen that the theory discussed in this paper becomes completely equivalent to the model of coupled dark matter and energy developed by Amendola [45]. Therefore one should expect that for our model the accelerating expansion would start at a maximum redshift of around $z \sim 5$ [45] in a way which is consistent with the type Ia supernovae Hubble diagram, including the farthest known supernova at $z=1.7$, while allowing for structure formation during the acceleration regime. In fact, if there was an epoch at $z_{a c c}$ in the past before which uncoupled baryons with critical density $\Omega_{b}$ dominated a decelerating flat universe filled also with tachyon fields, then in the present theory with $\gamma$ given by Eq. (5.6) that epoch would be given by

$$
1+z_{\mathrm{acc}}=\left[-(1+3 \omega)\left(\frac{1-\Omega_{b}}{\Omega_{b}}\right)\right]^{1 /(3 \omega)},
$$

instead of the currently assumed value [45]

$$
z_{\mathrm{acc}}=\left[-(1+3 \omega)\left(\frac{\Omega_{\phi}}{\Omega_{D M}+\Omega_{b}}\right)\right]^{1 /(3 \omega)} \sim 1 .
$$

Finally, according to Eq. (5.7) for the allowed values of $\Omega_{b}$, on the phantom regime $(\omega<-1)$ the larger $|\omega|$ the smaller $z_{\text {acc }}$.

5 Thus, since the nature and properties of nonrotating, chargeless black holes of any sizes do not qualitatively distinguish the original characteristics of the stuff out of which they were formed, the difficulties encountered so far to justify presently considered mechanisms describing how central SMBHs were formed within galaxy bulges [46] might all be circumvented if we conjectured that, since it turns out that the tachyon field perturbations in density con- 
trast took place with a spatial distribution nearly matching that of matter fluctuations, and they initially may rapidly have grown during a more or less long period, shortly after the onset of tachyon dark-energy domination at a sufficiently large $z$, giant black holes mainly made up of dark matter and energy, formed by the kind of quickly accelerated collapsing procedure considered in Sec. IV, started forming at the galactic centers and very rapidly reached a state where they were able to switch on stars in the surrounding bulge by means of a process similar to the Silk-Rees mechanism [42]. That conjecture would seem to decide the debate on which came first, the black hole or the galaxy, in favor of the latter, but required the existence of galaxies devoid of high star densities until their central SMBHs were formed.

As to the influence that ordinary matter sources may have on the stability of tachyon dark energy and its accelerated collapse, we note that if these sources contribute as a perturbation, one can analyze the whole model via phase space [47]. Then the potentials (2.10) and (2.16) and the solutions (2.8) and (2.13) would correspond to dynamical attractors for, respectively, tachyon dark and phantom energy domination with their respective cosmological effects. For these attractors we have [47] $\Theta=$ $V V^{\prime \prime} /\left(V^{\prime}\right)^{2}=3 / 2$, at critical points defined by $8 \pi G \lambda_{c}$ which equals $3 \sqrt{1+\omega} / T$ for dark energy and $-3 \sqrt{|\omega|}-1 / T$ for phantom energy. It follows then that: (i) the models of Secs. II, III, and IV are stable to the presence of small proportions of ordinary matter, and (ii) even though that ordinary matter would roughly follow distribution of the dark matter and energy, one would expect it not to significantly undergo initial accelerated collapse as it had to obey a different equation of state. Thus, ordinary baryonic matter would essentially remain as the stuff out of which stars are going to be formed.

\section{CONCLUSIONS AND FURTHER COMMENTS}

The assumed dark energy content of the Universe possibly reflects the greatest unsolved problem of all of physics. This paper has explored some potentially important observational consequences from one of the most promising models suggested to describe dark energy, the so-called tachyon model. Thus, by using tachyonlike theories whose starting Lagrangians generally are inspired by string theories, we have investigated the properties of general FRW cosmological solutions that are fully consistent with the whole dynamical structure of the theories. Assuming a general equation of state $p=\omega \rho$, such so- lutions describe accelerating universes in the interval $-1 / 3>\omega>-1$ and, among other weird properties characterizing the phantom regime, all show a big rip singularity in finite time when $\omega<-1$. It has been generally shown as well that the entropy of a universe filled with phantom energy is always positive, though its temperature becomes definite negative. This keeps still phantom cosmology as a real, observationally not excluded possibility.

Fluctuations taking place in both of such regimes have been studied by using a synchronous perturbation formalism and the relativistic analog of the Newtonian first order perturbation theory. In both formalisms the result has been obtained that the considered tachyon theory is stable to fluctuations along the entire considered interval of the state equation parameter $-5 / 3<\omega=p / \rho<-1 / 3$.

A potential problem with the tachyon models considered in this paper stems from the imaginary value of the sound speed. In fact a value $c_{s}^{2}<0$ implies occurrence of instability on scales below the Jeans limit for scalar field fluctuations [20] which may grow therefore exponentially. Whereas when these models are considered as pure cosmic vacuum components this could in fact be regarded as an actual problem, if the tachyon models are viewed as the sum of two components [17], one describing the negative pressure vacuum stuff and the other describing the dustlike cold dark matter contributing $\Omega_{m} \sim 0.35$ and clustering gravitationally at small scales, the results obtained in this paper for a negative value for $c_{s}^{2}$ might instead be regarded as originating an accelerated process of gravitational collapse inexorably leading to the rapid formation of black holes in a way that may explain some recent observations in galaxies and superclusters that concern gravitationally collapsed objects such as supermassive black holes. Alcaniz and Lima suggested nevertheless that a quintessence component pushes back to higher redshift the epoch of formation of some galaxies [48]. The analysis carried out by these authors is based however on a simple dark-energy component which is uncoupled to dark matter. If one assumes that dark matter and energy are coupled to each other, then since it turns out that the perturbations in density contrast may initially grow during a period, we have in fact advanced the conjecture that supermassive black holes with masses ranging from $10^{6}$ to $10^{9}$ solar masses which have been observed at the center of most galactic bulges could actually be originated by the rapid accelerated collapse of the gravitationally enlarged overdense regions made up of tachyon dark matter and energy. Rather than being destructive influences on their surroundings, the resulting giant black holes would then have a very creative impact in the formation of the host galaxies. This would offer a novel alternate resolution to the problem of supermassive 
black hole formation. Much calculation and observational scrutiny is however needed before such a conjecture can be seriously considered. In particular, it appears that future observations on supernovae of type Ia at high redshift will be decisive in order to check acceptability of our conjecture.

\section{ACKNOWLEDGMENTS}

The author thanks Carmen L. Sigüenza for useful discussions and Yun-Song Piao, Fabio Finelli, J.S. Alcaniz, and J. A. S. Lima for valuable correspondence. This work was supported by DGICYT under Research Project No. BMF2002-03758.
[1] S. Perlmutter et al., Astrophys. J. 483, 565 (1997); S. Perlmutter et al., Nature (London) 391, 51 (1998); P. M. Garnavich et al., Astrophys. J. Lett. 493, L53 (1998); B. P. Schmidt, Astrophys. J. 507, 46 (1998); A. G. Riess et al., Astron. J. 116, 1009 (1998).

[2] M. S. Turner, astro-ph/0108103; M. Turner and A. G. Riess, Astrophys. J. 569, 18 (2002).

[3] B. Ratra and P. J. E. Peebles, Rev. Mod. Phys. 75, 559 (2002).

[4] C. Wetterich, Nucl. Phys. B 302, 645 (1988); B. Ratra and P. J. E. Peebles, Astrophys. J. 325, L17 (1988).

[5] C. Armendáriz-Picón, T. Damour, and V. Mukhanov, Phys. Lett. B 458, 209 (1999); J. Garriga and V. Mukhanov, Phys. Lett. B 458, 219 (1999); T. Chiba, T. Okabe, and M. Yamaguchi, Phys. Rev. D 62023511 (2000); C. Amendáriz-Picón, V. Mukhanov, and P. J. Steinhardt, Phys. Rev. Lett. 854438 (2000); C. Amendáriz-Picón, V. Mukhanov, and P. J. Steinhardt, Phys. Rev. D 63, 103510 (2001); L. P. Chimento and A. Feinstein, Mod. Phys. Lett. A 19, 761 (2004).

[6] J. C. Jackson and M. Dodgson, Mon. Not. R. Astron. Soc. 297, 923 (1998); J. C. Jackson, Mon. Not. R. Astron. Soc. 296, 619 (1998); R. R. Caldwell, R. Dave, and P. J. Steinhardt, Phys. Rev. Lett. 80, 1582 (1998); L. Wang and P. J. Steinhardt, Astrophys. J. 508, 483 (1998); R. R. Caldwell and P. J. Steinhardt, Phys. Rev. D 57, 6057 (1998); G. Huey, L. Wang, R. Dave, R. R. Caldwell, and P. J. Steinhardt, Phys. Rev. D 59, 063005 (1999); P. F. González-Díaz, Phys. Rev. D 62, 023513 (2000).

[7] P. J. Steinhardt, L. Wang, and I. Zlatev, Phys. Rev. Lett. 82, 896 (1999); P. J. Steinhardt, L. Wang, and I. Zlatev, Phys. Rev. D 59, 123504 (1999); I. Zlatev and P. J. Steinhardt, Phys. Lett. B 459, 570 (1999).

[8] A. Kamenshchik, U. Moschella, and V. Pasquier, Phys. Lett. B 511, 265 (2001); N. Bilic, G. B. Tupper, and R. Viollier, Phys. Lett. B 535, 17 (2001); M. C. Bento, O. Bertolami, and A. A. Sen, Phys. Rev. D 66, 043507 (2002).

[9] A. Sen, J. High Energy Phys. 04 (2002) 048; A. Sen, Mod. Phys. Lett. A 17, 1797 (2002); G.W. Gibbons, Phys. Lett. B 537, 1 (2002).

[10] A. C. Baccigalupi, A. Balbi, S. Matarrase, F. Perrotta, and N. Vittorio, Phys. Rev. D 65, 063520 (2002); M. Melchiorri, L. Mersini, C. J. Odman, and M. Tradden, Phys. Rev. D 68, 043509 (2003); M. Doupis, A. Riazuelo, Y. Zolnierowski, and A. Blanchard, Astron. Astrophys. 405, 409 (2003); L. Tonry et al., Astrophys. J. 594, 1 (2003); J. S. Alcaniz, Phys. Rev. D 69, 083521 (2004).
[11] R. R. Caldwell, Phys. Lett. B 545, 23 (2002).

[12] B. McInnes, J. High Energy Phys. 08 (2002) 029; G.W. Gibbons, hep-th/0302199; A. E. Schulz and M. J. White, Phys. Rev. D 64, 043514 (2001); J. G. Hao and X. Z. Li, Phys. Rev. D 67, 107303 (2003); S. Nojiri and S. D. Odintsov, Phys. Lett. B 562, 147 (2003); 565, 1 (2003); Phys. Lett. B571, 1 (2003); P.Singh, M. Sami, and N. Dadhich, Phys. Rev. D 68, 023522 (2003); J. G. Hao and X. Z. Li, Phys. Rev. D 68, 043501 (2003); 68, 083514 (2003); X. Z. Li and J. G. Hao, Phys. Rev. D 69, 107303 (2004); M. P. Dabrowski, T. Stachowiak, and M. Szydlowski, Phys. Rev. D 68, 067301 (2003); M. Szydlowski, W. Zaja, and A. Krawiec, astro-ph/ 0401293; E. Elizalde and J. Quiroga H., Mod. Phys. Lett. A 19, 29 (2004); V. B. Johri, astro-ph/0311293 [Phys. Rev. D (to be published)]; L. P. Chimento and R. Lazkoz, Phys. Rev. Lett. 91, 211301 (2003); H. Q. Lu, hep-th/0312082; M. Sami and A. Toporensky, Mod. Phys. Lett. A 19, 1509 (2004); R. Naboulsi, gr-qc/0303007; J. M. Cline, S. Jeon, and G. D. Moore, hep-ph/0311312 [Phys. Rev. D (to be published)]; X. H. Meng and P. Wang, hep-ph/0311070; H. Stefancic, Phys. Lett. B 586, 5 (2004); H. Stefancic, astro-ph/0312484, [Eur. Phys. J. C (to be published); D. J. Liu and X. Z. Li, Phys. Rev. D 68, 067301 (2003); A. Yurov, astro-ph/0305019; Y. S. Piao and E. Zhou, Phys. Rev. D 68, 083515 (2003); Y. S. Piao and Y.Z. Zhang, astro-ph/0401231 [Phys. Rev. D (to be published)]; H. Q. Lu, hep-th/0312082; L. P. Chimento and R. Lazkoz, astro-ph/0405518; J. M. Aguirregabiria, L. P. Chimento, and R. Lazkoz, Phys. Rev. D 70, 023509 (2004); J. D. Barrow, Class. Quant. Grav. 21, L79 (2004); S. Nojiri and S. D. Odintsov, hep-th/0405078; E. Elizalde, S. Nojiri, and S. D. Odintsov, hep-th/0405034.

[13] P. F. González-Díaz, Phys. Rev. D 68, 084016 (2003).

[14] R. R. Caldwell, M. Kamionkowski, and N. N. Weinberg, Phys. Rev. Lett. 91, 071301 (2003).

[15] P. F. González-Díaz, Phys. Lett. B 586, 1 (2004).

[16] P. F. González-Díaz, Phys. Rev. D 68, 021303(R) (2003); M. Bouhmadi and J. A. Jiménez-Madrid, astro-ph/ 0404540 .

[17] T. Padmanabhan, Phys. Rev. D 66, 021301 (2002); T. Padmanabhan and T. R. Choudhury, Phys. Rev. D 66, 081301 (2002); J.S. Bagla, H. K. Jassal, and T. Padmanabhan, Phys. Rev. D 67, 063504 (2003).

[18] P. F. González-Díaz, Phys. Rev. D 69, 103512 (2004).

[19] L. R.W. Abramo and F. Finelli, Phys. Lett. B 575, 165 (2003). 
[20] D. Carturan and F. Finelli, Phys. Rev. D 68, 103501 (2003); H. B. Sandvik, M. Tegmark, M. Zaldarriaga, and I. Waga, Phys. Rev. D 69, 123524 (2004); A. Frolov, L. Kofman, and A. Starobinsky, Phys. Lett. B 545, 8 (2002).

[21] L. R.W. Abramo, F. Finelli, and T. S. Pereira, astro-ph/ 0405041 [Phys. Rev. D (to be published)].

[22] L. Amendola, F. Finelli, C. Burigana, and D. Carturan, J. Cosmol. Astropart. Phys. 07 (2003) 005.

[23] M. J. Rees, in Black Holes and Relativistic Stars, edited by R. M. Wald (Chicago University Press, Chicago, 1988); M. J. Rees, in Black Holes in Binaries and Galactic Nuclei, edited by L. Kaper, E. P. J. van den Heurel, and P. A. Would (Springer-Verlag, New York, 2001); F. D. Machetto, in Towards a New Millennium in Galaxy Morphology, edited by D. L. Block et al., (Kluwer, Dordrecht, 1999).

[24] P.T. P. Viana and A. R. Liddle, Phys. Rev. D 57, 674 (1998); W. Hu, Astrophys. J. 506, 495 (1998); M. White, Astrophys. J. 506, 485 (1998).

[25] P. F. González-Díaz, Phys. Rev. D 69, 063522 (2004).

[26] I. Brevik, S. Nojiri, S. D. Odintsov, and L. Vanzo, Phys. Rev. D 70, 043520 (2004); J. A. S. Lima and J. S. Alcaniz , astro-ph/0402265.

[27] D. Youm, Phys. Lett. B 531, 276 (2002); E. Verlinde, hepth/0008140; J. L. Cardy, Nucl. Phys. B 270, 186 (1986).

[28] J. B. Hartle and S.W. Hawking, Phys. Rev. D 13, 2188 (1976).

[29] E. M. Lifshitz and I. Khalatnikov, Adv. Phys. 12, 185 (1963); L. P. Grishchuk, Phys. Rev. D 50, 7154 (1994); J. C. Fabris and J. Martin, Phys. Rev. D 55, 5205 (1997).

[30] J. P. E. Peebles, The Large Scale Structure of the Universe (Cambridge University Press, Cambridge, 1980).

[31] T. Padmanabhan, Structure Formation in the Universe (Cambridge University Press, Cambridge, 1993).
[32] M. Abramowitz and I. A. Stegun, Handbook of Mathematical Functions (Dover, New York, 1965).

[33] P. F. González-Díaz, Phys. Rev. Lett. 93, 071301 (2004).

[34] P. Fosalba and E. Gaztañaga, Mon. Not. R. Astron. Soc. 31, 535 (1998).

[35] M. G. Haehnelt, in Coevolution of Black Holes and Galaxy, Carnegie Observatories Series Vol. 1, edited by L.C. Ho (Cambridge University Press, Cambridge, 2003).

[36] C. van de Bruck, Astron. Astrophys. 421, 71 (2004).

[37] D. Lynden-Bell, Nature (London) 223, 690 (1969).

[38] S. L. Shapiro, in Coevolution of Black Holes and Galaxies, Carnegie Observatories Astrophysical Series Vol. 1, edited by L. C. Ho (Cambridge University Press, Cambridge, 2003); C. Tyler, B. Janus, and D. SantosNoble, astro-ph/0309008.

[39] R. P. van der Marel, J. Gerssen, P. Guhathakurta, R. C. Peterson, and K. Gebhardt, astro-ph/0209314 [Astron. J. (to be published)]; K. Gebhardt, R. M. Rich, and L. C. Ho, Astrophys. J. 578, L41 (2002).

[40] C. C. Steidel, M. Giavalisco, M. Pettini, M. Dickinson, and K. L. Adelberger, Astrophys. J. 462, L17 (1996).

[41] K. Bebhardt et al., Astrophys. J. 539, L13 (2000); L. Farrarese and D. Merritt, Astrophys. J. 539, L9 (2000).

[42] J. Silk and M. J. Rees, Astron. Astrophys. 331, 1 (1998); M. G. Haehnelt, P. Natarajan, and M. J. Rees, Mon. Not. R. Astron. Soc. 3000, 817 (1998).

[43] A. G. Riess et al., Astrophys. J. 560, 49 (2001); A. G. Riess et al., astro-ph/0402512 [Astrophys. J. (to be published)].

[44] H. Martel, Astrophys. J. 421, L67 (1994).

[45] L. Amendola, Mon. Not. R. Astron. Soc. 342, 221 (2003).

[46] J. Silk, Space Sci. Rev. 100, 41 (2002).

[47] J. G. Hao and X. Z. Li, astro-ph/0309746.

[48] J. S. Alcaniz and J. A. S. Lima, Astrophys. J. 550, L133 (2001). 